\title{
Retour sur les critiques anarchistes du marxisme
}

Poser la question de la pertinence contemporaine des critiques anarchistes du marxisme ${ }^{1}$, c'est peutêtre d'abord se demander ce que nous pouvons en faire à une époque comme la nôtre. Il n'aura échappé à personne que nous vivons au $\mathrm{XXI}^{\mathrm{e}}$ siècle. Ce que E. Hobsbawm appelle le court $\mathrm{XX}^{\mathrm{e}}$ siècle $^{2}$, et qui a commencé avec la première guerre mondiale avant de s'achever avec la dislocation de l'empire dit soviétique autour de 1990, est étroitement lié aux aventures et surtout aux mésaventures du marxisme puisque cette période correspond exactement à ce qu'a duré le premier régime politique à s'être réclamé explicitement de Marx. Or l'analyse économique, sociale et politique ainsi que le type d'émancipation promus par le marxisme sont ressortis de cette période, à tort ou à raison, durablement discrédités. De sorte que se demander quelle est l'actualité des critiques anarchistes du marxisme, c'est aussi se demander si ces critiques ne risquent pas de partager le sort de ce dernier. La promotion du marxisme, au cours du $\mathrm{XX}^{\mathrm{e}}$ siècle, au rang de doctrine officielle, de doctrine d'État a, dans les faits, conduit pour lui à une double défaite : transformé en doctrine d'État et de parti, ce qui a conduit à une profonde altération de son contenu et de sa visée émancipatrice, il a partagé le sort des États et des mouvements politiques auquel il avait été associé. Pour les anarchistes, qui furent les premiers à la combattre, cette évolution pourrait bien s'apparenter à une amère victoire.

L'oubli du marxisme et de sa critique dans les formes contemporaines de l'anarchisme

En forçant à peine le trait, on peut présenter de la manière suivante ce que fut la réaction de nombre de militants et de penseurs libertaires (et de marxistes hétérodoxes) aux événements qui ont marqué l'effondrement des régimes d'Europe de l'est à la fin $\mathrm{du} \mathrm{XX}^{\mathrm{e}}$ siècle. La fin du prétendu socialisme réel signifiait pour beaucoup qu'une parenthèse malheureuse se refermait enfin, celle qui avait vu précisément le projet émancipateur porté depuis le milieu du $\mathrm{XIX}^{\mathrm{e}}$ siècle par le mouvement ouvrier être falsifié, identifié au seul marxisme, et à une version bien particulière de ce dernier, et pour finir à la cause de quelques États et des oligarchies qui les dominaient. Autrement dit, la fin de l'Union dite soviétique (qui avait précisément pris le nom du mode d'organisation sur les ruines duquel elle s'était édifiée ${ }^{3}$ ) n'était-elle pas l'occasion de rouvrir tous ces possibles que la révolution russe et son destin catastrophique avaient brutalement fermés ? Et en particulier, n'était-ce pas l'occasion de faire ressortir, de sous la chape de plomb de l'histoire, les potentialités émancipatrices du mouvement libertaire qui s'était précisément signalé par son opposition aussi bien à l'ordre capitaliste qu'au socialisme autoritaire? Littéralement, si l'on me passe la plaisanterie, n'était-on pas reparti comme en 14 ?

Cette perception avait, à l'évidence, quelque chose de naïf et de déraisonnablement optimiste. Elle passait outre le fait que la défaite du marxisme avait été précédée par celle de mouvements qui, pour n'avoir (peut-être) pas perdu leur âme, n'en avaient pas moins à s'interroger sur leur propre faillite (que l'on songe par exemple à la défaite de l'anarchisme espagnol en 1937). Elle sous-estimait en outre le fait que ce discrédit jeté sur le marxisme avait, plus largement, touché tout projet révolutionnaire, toute perspective de remise en cause radicale de l'ordre économique, social et politique dominant, au nom du fameux adage selon lequel « on voit bien où tout cela mène ». La vision de l'histoire qui consiste à faire $\mathrm{du} \mathrm{XX}^{\mathrm{e}}$ siècle une parenthèse malheureuse où le mouvement révolutionnaire a fait fausse route, s'est retrouvé dans une impasse dont il pourrait enfin sortir, en quelque sorte en rebroussant chemin, doit néanmoins être mentionnée parce qu'elle contribue à l'intelligence de la résurgence contemporaine de différentes formes d'anarchisme, qui s'était déjà donnée à voir lorsque les événements des années 68 avaient ébranlé les marxismes orthodoxes (c'est-à-dire prétendant à l'orthodoxie) et les organisations qui s'en réclamaient. Cette résurgence a

1 Une première version de ce texte a été lue lors du colloque organisé à Lyon le 8 mars 2013 sur «L'actualité de Marx ». Je remercie les étudiants qui avaient organisé cette journée pour leur invitation et leur écoute attentive.

2 Eric Hobsbawm, L'Âge des extrêmes, histoire du court XXe siècle, Bruxelles, Complexe, 1999.

3 Cette critique fut formulée très tôt par quelques-uns des derniers représentants de l'anarchisme " classique », notamment en 1921 par Rudolf Rocker dans Les Soviets trahis par les bolcheviks, Paris, Spartacus, 1998. 
pu être observée dans les luttes sociales, qu'il s'agisse du contournement des appareils syndicaux dans les luttes officielles (pratique des coordinations et des collectifs de lutte), ou de pratiques d'auto-organisation et d'action directe par exemple dans des luttes de travailleurs précaires (et plus largement dans ce qu'on appelait, à la fin des années 90, les « luttes des sans » : sans-papiers, sanslogis, sans-travail), avec dans les deux cas une nouvelle manière de répondre à la question de l'autoémancipation, posée depuis les origines du mouvement ouvrier. Elle a pu aussi être observée à l'occasion du mouvement altermondialiste des années 2000, à la fois au travers de formes de lutte se revendiquant d'une horizontalité dans l'organisation et dans la promotion d'alternatives non-étatistes au capitalisme mondialisé ${ }^{4}$. Elle s'est également manifestée, en France, dans des formes radicales de contestation telles celles qui ont marqué un mouvement comme celui dit « du CPE » en 2006. Elle a enfin eu sa répercussion, inévitablement, dans l'éveil d'un certain intérêt du monde académique pour le mouvement anarchiste, son histoire, ses théories et son actualité 5

À cette occasion, les observateurs du mouvement anarchiste, qui en sont aussi parfois (mais pas toujours) des participants, ont tenté de qualifier de diverses manières ce qu'ils considéraient comme certains de ses traits nouveaux. On a pu ainsi parler d'anarchisme hors les murs ${ }^{6}$, notamment pour désigner un anarchisme de la pratique, en dehors des organisations, et qui parfois ne se reconnaît pas comme tel. On a encore choisi de qualifier les nouvelles formes prises par le mouvement de néo-anarchisme ${ }^{7}$, désignant par là un anarchisme qui n'est pas en continuité stricte avec l'anarchisme historique et rompt avec certains de ses postulats, par exemple celui de la révolution sociale. On a vu enfin apparaître un post-anarchisme ${ }^{8}$, courant strictement universitaire partant de l'hypothèse que l'anarchisme historique partagerait avec la modernité un certain nombre de postulats avec lesquels il s'agirait de rompre. Dans ces reconfigurations contemporaines de l'anarchisme, ce qui est mis en question, c'est la possibilité de faire une histoire continue de l'anarchisme à la fois comme théorie politique (ou antipolitique) et comme ensemble de pratiques antiautoritaires, puisque l'anarchisme pratique semble oublier l'histoire, et que le post-anarchisme semble cruellement coupé de toute pratique militante.

Or dans les différentes formes contemporaines de l'anarchisme (en y incluant pour une part celles qui sont héritées de l'anarchisme historique, les formes organisées, fédératives ou anarchosyndicalistes), il existe une tendance à traiter le marxisme "en chien crevé », pour employer une formule que Marx lui-même avait utilisée pour qualifier l'attitude de certains auteurs de son temps à l'encontre de Hegel$^{9}$. "Traiter le marxisme en chien crevé », c'est bien pire que d'en proposer la critique la plus dévastatrice qui soit, par exemple en s'en prenant à ses postulats que l'on considère comme les plus fondamentaux, c'est considérer que le marxisme ne vaut même plus la peine qu'on le critique, qu'on le lise, et encore moins qu'on cherche chez lui quoi que ce soit qui augmente notre puissance d'agir. C'est faire de Marx une « ruine du temps » dont il faudrait « se détourner » (pour reprendre une formule que le jeune Engels employait à propos du vieux Schelling ${ }^{10}$ ), sous peine précisément de perdre son temps.

4 C'est notamment de cette actualité que rend compte l'ouvrage de Uri Gordon, Anarchy alive! Les politiques antiautoritaires de la pratique à la théorie, trad. Vivien Garcia, Lyon, ACL, 2012.

5 On en trouve des illustrations notamment dans la revue anglophone Anarchist Studies et dans l'ouvrage que j'ai coordonné avec Daniel Colson et Mimmo Pucciarelli, Philosophie de l'anarchie, Lyon, ACL, 2012.

6 Voir la mise au point de Uri Gordon dans Anarchy alive !, op. cit., pp. 30-33.

7 Cette désignation a été proposée par Tomás Ibañez. Voir notamment de cet auteur «L'anarchisme est un type d'être constitutivement changeant. Arguments pour un néo-anarchisme », in J.-C. Angaut, D. Colson et M. Pucciarelli, Philosophie de l'anarchie, op. cit., pp. 357-372.

8 Le principal représentant de ce courant peu militant est Saul Newman, From Bakunin to Lacan: AntiAuthoritarianism and the Dislocation of Power, Lexington Books, 2001. Pour un exposé et une critique des théories post-anarchistes, voir Vivien Garcia, L'Anarchisme aujourd'hui, Paris, L'Harmattan, 2007.

9 K. Marx, Postface du deuxième tirage du Capital, t. I, Paris, Éditions Sociales, 1967, p. 29. Dans ce texte, Marx reprend cette formule à la préface de l'Encyclopédie de Hegel, qui se référait à la manière dont, selon Lessing, Spinoza était désormais traité.

10 F. Engels, Schelling und die Offenbarung, in Marx-Engels Gesamtausgabe, I/3, Berlin, 1985, p. 311. 
Au rebours de cette tendance des anarchismes contemporains, il semble important de souligner que l'anarchisme perd quelque chose à réserver ce traitement à Marx, non seulement parce qu'il est susceptible de trouver encore dans l'arsenal marxien des armes de résistance à la domination qui ne sont pas toutes de vieux tromblons, mais aussi parce que la critique anarchiste de Marx et du marxisme peut encore apporter quelque chose à l'anarchisme contemporain, qu'il s'agisse de lutter contre la résurgence de formes autoritaire de marxisme, d'utiliser des armes forgées à l'occasion de cette critique contre d'autres éléments de domination ou d'interroger quelques postulats qui soustendent certaines formes d'activisme anarchiste.

On peut, pour commencer, et en revisitant à grands traits l'histoire des rapports entre marxisme et anarchisme, montrer d'une part que l'attitude de rejet de Marx chez la plupart des anarchistes contemporains, si elle n'est pas toujours raisonnable, a néanmoins ses raisons historiques, et d'autre part qu'au rebours de l'ignorance réciproque dans laquelle marxisme et anarchisme sont largement demeurés l'un vis-à-vis de l'autre, il a existé des tentatives théoriques et pratiques de penser à la fois une appropriation anarchiste de Marx et une appropriation marxiste de l'anarchisme.

L'anarchisme comme anti-marxisme

Si l'on cherche d'abord les raisons historiques pour lesquelles anarchisme et marxisme sont en grande partie demeurés dans une forme d'ignorance mutuelle, et en tout cas pour quelles raisons aujourd'hui la plupart des militants et des théoriciens de ces deux courants entretiennent des relations de méconnaissance réciproque, un retour aux origines s'impose ${ }^{11}$. L'anarchisme est originellement un anti-marxisme. Il faut entendre par là que l'acte de naissance historique de l'anarchisme en tant que mouvement ${ }^{12}$, c'est la scission intervenue au sein de l'Association Internationale des Travailleurs (que l'on a coutume de désigner comme la Première Internationale), à l'issue de ce qu'il est convenu d'appeler le conflit entre Marx et Bakounine - bien que ce conflit n'ait fait qu'incarner un conflit plus profond au sein du mouvement ouvrier naissant autour de la question de la politique et de l'organisation et que, bien souvent, Bakounine n'ait fait que formuler des critiques qui avaient été élaborées collectivement ${ }^{13}$.

Le motif de ce conflit, tel qu'il se donne à voir dans les textes où Bakounine critique Marx ${ }^{14}$, est en effet le suivant : Bakounine, qui ne connaît de Marx que sa personne (qu'il goûte assez peu) et le Manifeste communiste, attribue à Marx l'intention de faire de l'Internationale une sorte de regroupement de partis politiques participant aux luttes électorales et ayant pour but de conquérir par ce biais le pouvoir au sein des nations où existe un système représentatif, ce qui impliquerait une feuille de route assez claire pour les prolétaires des différents pays : parvenir à un régime représentatif, conquérir le suffrage universel, s'organiser en parti, gagner les élections ${ }^{15}$. C'est ainsi qu'il interprète la politique des socialistes allemands et l'idée, exprimée dans le Manifeste, selon laquelle la classe ouvrière doit se rendre maîtresse de l'appareil d'État pour le faire fonctionner à son profit, faire disparaître les oppositions de classe par l'extinction de la bourgeoisie en tant que

11 Ce retour aux origines n'exclut évidemment pas que l'on mentionne l'histoire ultérieure, qui a contribué à ce que le différend initial se cristallise en deux camps : l'écrasement du soulèvement de Cronstadt en 1921 et plus largement la répression croissante des anarchistes en Russie après 1918, la manière dont l'armée rouge a utilisé le soulèvement anarchiste de Makhno en Ukraine pour vaincre les armées blanches avant de se retourner contre lui, la liquidation de la révolution espagnole (et de nombre de révolutionnaires) par Staline en 1936-37, pour ne citer que les événements les plus connus.

12 Notamment si l'on estime cet acte de naissance est l'Internationale anti-autoritaire qui se réunit pour la première fois à Saint-Imier en septembre 1872 .

13 Voir sur ce point Mathieu Léonard, L'Émancipation des travailleurs. Une histoire de la Première Internationale, Paris, La Fabrique, 2011.

14 Ces textes sont principalement rassemblés dans le volume III des Euvres complètes de Bakounine, Paris, Champ Libre, 1974-1982.

15 Voir par exemple le manuscrit de mars 1872 «L'Allemagne et le communisme d'État» dans lequel Bakounine attaque « le Parti de la démocratie socialiste, fondé en Allemagne par les disciples de Marx, Lassalle d'abord, puis Liebknecht et Cie, le parti qui recommande aux ouvriers de l'Allemagne de chercher leur émancipation au moyen de la transformation de l'État actuel en un État populaire » (Euvres complètes, op. cit., vol. III, p. 113). 
classe, avant finalement de disparaître - puisque la raison d'être de l'État n'est rien d'autre que la perpétuation de la domination de classe, une société sans classes (c'est-à-dire en l'occurrence qui n'est plus régie par la division entre propriétaire des moyens de production et propriétaires de leur seule force de travail) serait une société sans État.

La contestation de cette thèse politique de Marx va conduire chez Bakounine à la contestation d'une thèse qui est de l'ordre la philosophie de l'histoire, à laquelle la première s'articule, selon laquelle l'histoire est guidée par le développement nécessaire du mode de production capitaliste vers l'aiguisement des luttes de classes et la concentration de plus en plus forte du capital, qui rend à la fois plus nécessaire et plus aisé le renversement de la domination de la bourgeoisie. À ce qu'il considère comme une approbation de la nécessité historique, Bakounine oppose l'idée selon laquelle, quand bien même les conditions de production et de reproduction de la vie humaine seraient déterminantes dans le développement de l'humanité, certaines des causes secondaires, comme l'État, peuvent acquérir une importance plus grande. C'est ce qui sous-tend, philosophiquement, la fameuse proposition sur l'abolition de l'héritage défendue par Bakounine au congrès de Bâle de l'AIT en 1869, et combattue par Marx et ses amis : ce droit peut bien n'être que le reflet juridique d'un fait économique, il n'en a pas moins une importance déterminante dans le maintien de ce dernier.

La critique adressée par Bakounine à la conception marxienne de l'histoire est donc double. D'une part, cette conception minorerait excessivement le rôle du facteur politique dans la marche de l'histoire. D'autre part, Marx sanctifierait la marche de l'histoire. Or pour Bakounine, qu'il y ait des tendances centralisatrices dans l'histoire ne signifie pas du tout que ces tendances soient objectivement révolutionnaires ; que la genèse du mode de production capitaliste et la centralisation étatique qui l'accompagne soient des tendances historiques fortes ne signifient pas pour autant qu'il faudrait les approuver, et quand bien même elles auraient un caractère inéluctable, on aurait raison de se révolter contre elles en tant qu'elles marquent un progrès dans l'assujettissement des êtres humains ${ }^{16}$. Dans cet esprit, le fait de résister à la mise en place de l'usine, ou à l'introduction de machines dans le processus de production, peut être interprété comme une forme de réaction de la part de tenants d'un mode de production en voie de disparition. Mais si l'on rompt avec la téléologie historique lourde qui est celle du marxisme, on peut aussi l'interpréter comme une défense de la liberté contre une forme inédite et redoutable d'oppression (et aussi comme une défense réussie des intérêts des ouvriers du secteur concerné). Il n'y a rien d'étonnant à ce que ce soit parmi les libertaires ${ }^{17}$ qu'on ait pu trouver des auteurs pour réhabiliter le mouvement luddite, que l'historiographie marxiste considérait comme rétrograde parce qu'il s'opposait au progrès technique, à la mise en place de grandes concentrations industrielles, et in fine à la possibilité d'organiser en masse la classe ouvrière.

S'agissant du statut de la politique, Bakounine considère que la saisie du pouvoir d'État par une organisation révolutionnaire inspiré par le programme (présumé) de Marx conduirait à l'émergence d'une nouvelle classe qui administrerait la capital arraché des mains de la bourgeoisie et opprimerait à son tour à la fois la classe ouvrière et la paysannerie ${ }^{18}$ - c'est souvent ce que l'on désigne, par une formule problématique, comme $l^{\prime}$ «analyse prophétique ${ }^{19}$ par Bakounine de la nécessaire dégénérescence de l'État ouvrier. À cette position politique qu'il attribue à Marx, Bakounine oppose

16 Dans le même texte, Bakounine écrit ainsi : «Matérialistes et déterministes comme M. Marx lui-même, nous aussi nous reconnaissons l'enchaînement fatal des faits économiques et politiques dans l'histoire [...] mais nous ne nous inclinons pas indifféremment devant eux, et surtout nous nous gardons bien de les louer et de les admirer lorsque, par leur nature, ils se montrent en opposition flagrante avec le but suprême de l'histoire, avec l'idéal foncièrement humain qu'on retrouve, sous des formes plus ou moins manifestes, dans les instincts, dans les aspirations populaires et sous les symboles religieux de toutes les époques. » (Euvres complètes, op. cit., vol. III, p. 195-196)

17 Voir notamment Julius Van Daal, La Colère de Ludd: la lutte des classes en Angleterre à l'aube de la révolution industrielle, Paris, L'Insomniaque, 2012.

18 Cette prédiction est notamment développée dans Étatisme et Anarchie (1873).

19 Voir par exemple Noam Chomsky, «Union soviétique et socialisme» in De l'espoir en l'avenir. Propos sur l'anarchisme et le socialisme, Marseille, Agone, 2001, p. 83. 
l'idée selon laquelle l'Internationale n'est pas un regroupement de partis ayant vocation à prendre le pouvoir par les urnes, mais un organe de systématisation et d'affirmation concrète de la solidarité objective, économique, qui lie le monde ouvrier. Au sein de cette organisation, explique encore Bakounine, il est naturel, et nécessaire, que différentes options politiques s'expriment, que ce soit celle de Marx, celle des amis de Blanqui (le coup d'État opéré par une petite minorité d'hommes décidés) ou celle de ce qu'il nomme le parti anarchique (l'insurrection généralisée qui anéantit l'État immédiatement, c'est-à-dire non pas instantanément, mais sans médiation), mais aucune des trois ne doit jamais devenir la position politique officielle de l'organisation, sous peine que celle-ci ne dégénère en un embryon d'État ${ }^{20}$.

On a là une critique de la séparation du politique et un idéal de résorption du politique dans l'économique qui seront repris, développés et transcrits en acte par le syndicalisme révolutionnaire à la fin du siècle et au début du suivant. Avec conséquence, Bakounine s'oppose également à toute forme de centralisation au sein de l'Internationale. Attribuant à Marx le projet de vouloir constituer le Conseil général de l'organisation en une sorte de directoire central, il prône la libre fédération des sections de l'organisation et la réduction de son organe central à un simple bureau de statistiques et de correspondance. C'est sur ce point (et non sur un programme anarchiste) que la plupart des sections de l'Internationale s'opposeront au Conseil général et quitteront l'organisation après l'exclusion de Bakounine au congrès de La Haye de $1872^{21}$.

La pertinence contemporaine d'une critique

Pour résumer, le conflit politique qui donne naissance historiquement à l'anarchisme lègue à ce dernier une triple critique dont il s'agit d'interroger la pertinence actuelle. En premier lieu une critique de la politique, celle-ci étant entendue comme une activité menée par des spécialistes et orientée vers la conquête du pouvoir par le biais des élections, à quoi est opposée une antipolitique (et non un apolitisme comme Marx et ses amis tendront à le prétendre), c'est-à-dire une activité relative à l'État, mais qui vise sa disparition ${ }^{22}$. En second lieu une critique des organisations comme source possible d'une nouvelle oppression, d'une nouvelle centralisation, d'une nouvelle bureaucratie, critique qui connaîtra un prolongement immédiat dans le syndicalisme révolutionnaire et chez les premiers auteurs qui souligneront la confiscation de la démocratie par les partis politiques $^{23}$. Enfin une critique de l'ordre de la philosophie de l'histoire en tant qu'est refusée à celleci tout télos qui l'orienterait, critique qui permet de comprendre pourquoi l'anarchisme, historiquement, n'a pas seulement survécu à ses défaites, mais qu'il les a transformées en légendes la Commune, Makhno, l'Espagne libertaire, etc. Ces critiques ne sont encore qu'esquissées chez Bakounine. S'agissant, des organisations, la position de Bakounine est plus complexe que ce qu'il dit de l'Internationale, puisqu'il est en même temps un grand amateur de sociétés secrètes, qu'il dote de règlements interminables, règlements qui sont loin de constituer l'esquisse d'une société débarrassée de toute forme de domination ${ }^{24}$. Quant à la philosophie de l'histoire, Bakounine adhère dans l'ensemble à la conception matérialiste de l'histoire telle qu'elle se donne à lire dans le Manifeste, il en rejette simplement le mécanisme grossier, sans rejeter une certaine téléologie. S'agissant de la politique, le conflit entre Marx et Bakounine donne lieu à un curieux chiasme, où

20 Voir tout particulièrement les premières pages de l'Écrit contre Marx, daté de la fin de l'année 1872, in CEuvres complètes, op. cit., vol. III, pp. 171-187.

21 C'est toutefois le Jurassien James Guillaume, et non Bakounine, qui imposa la tactique minimaliste conduisant finalement à l'isolement des marxistes et des blanquistes sur la question de l'autonomie des sections au sein de l'organisation : voir sur ce point l'introduction d'Arthur Lehning au vol. III des Euvres complètes de Bakounine, op. cit.

22 Cet antipolitisme est commun à Bakounine et Proudhon qui écrivait dans ses Carnets en 1852 : «Je fais de la politique pour la tuer et en finir avec la politique. » (Cité par Philippe Chanial, « Justice et contrat dans la république des associations de Proudhon », Corpus, $\mathrm{n}^{\circ}$ 47, 2004, p. 113.)

23 Moisei Ostrogorski, La Démocratie et les partis politiques (1902-1912), Paris, Fayard, 1993. Robert Michels, Zur Soziologie des Parteiwesens, Leipzig, Klinkhardt, 1911.

24 Je renvoie à la prochaine édition du Catéchisme révolutionnaire de Bakounine, Montreuil, Le Chat Ivre, 2014, édition qui comprendra le plus important des règlements de société secrète rédigé par le révolutionnaire russe. 
l'auteur qui est censé minorer le rôle des facteurs politiques dans l'histoire se voit accusé de faire de la saisie du pouvoir d'État l'objectif essentiel, et où l'auteur qui insiste sur le rôle des facteurs politiques et juridiques dans le développement du mode de production capitaliste (par exemple au travers de l'héritage) tourne le dos à toute activité politique séparée ou spécifique.

À ces trois critiques, il faut en ajouter une quatrième, qui fut d'abord propre à Bakounine en raison de son intérêt pour les pays n'ayant pas développé d'industrie. Comme une partie du marxisme après lui, Bakounine pointe chez Marx une identification de la classe ouvrière à une classe révolutionnaire par essence, ce qui conduit à rejeter comme potentiellement contre-révolutionnaires des éléments de la population comme le Lumpenproletariat, la petite paysannerie ou les jeunes déclassés. Lorsqu'il cherche à penser les conditions d'une révolution dans des pays où l'industrie et la classe ouvrière en sont encore à leurs balbutiements (Russie, mais aussi Italie), Bakounine n'emprunte pas la voie qui consisterait à dire que ces pays doivent d'abord se doter d'une classe ouvrière disciplinée par l'industrie pour pouvoir envisager une révolution sociale, il souligne plutôt le rôle qui revient aux masses paysannes ${ }^{25}$ et à la jeunesse ${ }^{26}$, voire à ce qui est décrié par Marx comme Lumpenproletariat $t^{27}$. On retrouvera, plus près de nous, chez Pierre Bourdieu, une analyse convergente du potentiel révolutionnaire que recèle l'existence $\mathrm{d}^{\prime}$ « intellectuels prolétaroïdes » qui ne sont pas parvenus à trouver leur place dans un champ déterminé et que « tout [...] incline à mettre au service de l'indignation et de la révolte populaires leurs capacités d'explicitation et de systématisation $»^{28}$.

Ces différentes critiques sont aussi intéressantes parce qu'elles entrent en résonance avec d'autres décentrements qui ont marqué l'époque contemporaine, en particulier lorsqu'il s'est agi de revisiter le problème de l'État. Il existe une parenté entre les critiques du marxisme formulées historiquement par les anarchistes et celles qui ont pu être présentées par des auteurs aussi différents que Michel Foucault et Pierre Bourdieu, notamment dans leur enseignement au Collège de France, lorsqu'ils ont eu respectivement à aborder la question de l'État ${ }^{29}$. On peut également signaler les prolongements qu'a trouvés la critique anarchiste d'une certaine philosophie marxiste de l'histoire chez les anthropologues libertaires Pierre Clastres $^{30}$ et David Graeber ${ }^{31}$.

Bien entendu, il faut apporter à cette présentation des critiques anarchistes de Marx plusieurs nuances. Tout d'abord, la position qui est attribuée par Bakounine et ses amis à Marx est fortement simplificatrice, et semble souvent s'appliquer aux conceptions des lassaliens et anticiper sur ce que sera le marxisme de la IIe Internationale. Sur la question politique, Marx a sans doute une position plus complexe, qui consiste à prendre en considération l'évolution propre à chaque pays, prônant dans certains cas l'organisation de la classe ouvrière en un parti destiné à prendre pacifiquement le pouvoir, mais n'excluant pas ailleurs que surviennent des révolutions. Surtout, Marx a oscillé, notamment au moment de la Commune de Paris, sur la question de la saisie du pouvoir d'État par le

25 C'est notamment le cas à la fin d'Étatisme et Anarchie, son dernier grand texte. Voir Euvres complètes, op. cit., vol. IV, p. 346.

26 Pour l'Italie, Bakounine souligne notamment le rôle révolutionnaire que pourrait jouer cette « grande masse de gens nés dans la classe bourgeoise, mais qui ayant dédaigné d'un côté le service de l'État, et n'ayant point trouvé de place ni dans l'industrie ni dans le commerce, se trouvent complètement déplacés et désorientés » (CEuvres complètes, op. cit., vol. I, p. 82). Voir pour la Russie le texte Quelques paroles à mes jeunes frères en Russie (septembre 1869) et son éloge de « le jeunesse déclassée et lettrée », in Le Socialisme libertaire, Paris, Denoël, 1973, p. 205.

27 Voir, dans la lettre à Netchaev du 2 juin 1870 l'incitation à se lier au « monde des vagabonds, des brigands et des voleurs » (CEuvres complètes, op. cit., vol. V, p. 234).

28 Pierre Bourdieu, «Le champ littéraire » (1982), Actes de la recherche en sciences sociales, vol. 89, septembre 1991, p. 32. Il semble donc difficile de soutenir, avec Daniel Bensaïd, que cette figure est identique à celle de l'intellectuel organique et « constitue une sorte de lumpen-intelligentsia ressentimentale » à laquelle « l'intellectuel authentique » s'oppose et que Bourdieu « récuse avec horreur» («Pierre Bourdieu, l'intellectuel et le politique », Contretemps, $\mathrm{n}^{\circ} 4$ (mai 2002), p. 165).

29 Michel Foucault, Naissance de la biopolitique (1979), Paris, Gallimard-Seuil, 2004. Pierre Bourdieu, Sur l'État (1989-1992), Paris, Seuil, 2012.

30 P. Clastres, La Société contre l'État, Paris, Minuit, 1972.

31 D. Graeber, Pour une anthropologie anarchiste, Montréal, Lux Éditeur, 2006. 
prolétariat : tout en continuant à suivre les efforts des socialistes allemands pour s'unir en un parti unique, Marx fait l'éloge des prolétaires parisiens qui ont compris que la classe ouvrière, dit-il corrigeant la formule du Manifeste, ne doit pas se contenter de prendre les pouvoirs de l'État tels qu'ils existent pour les faire fonctionner à son profit, mais doit immédiatement les détruire. S'agissant de l'organisation, Marx ne méprise absolument pas les formes d'organisation fondées sur la solidarité économique, il estime même qu'elles sont le lieu d'une préfiguration de la société sans classe, l'endroit où les prolétaires peuvent faire l'expérience d'un auto-gouvernement ${ }^{32}$. Enfin, si lorsqu'il formule de grandes tendances historiques, ou quelque chose comme des lois de l'histoire (typiquement dans l'avant-dernier chapitre du livre I du Capital à propos de la tendance historique de l'accumulation capitaliste), il est difficile de ne pas considérer chez lui une forme de téléologie historique, il en va souvent autrement dans les analyses qu'il propose de conjonctures historiques particulières. Ces nuances sont à la fois importantes et dérisoires : dérisoires parce qu'elles seront souvent gommées par les versions dominantes du marxisme, mais importantes parce que c'est précisément sur elles que pourront s'appuyer certains auteurs et militants pour proposer un rapprochement entre marxisme et anarchisme.

Il faut ensuite souligner que cette hostilité réciproque entre partisans de Marx et partisans de Bakounine, même si elle était nourrie de méconnaissance réciproque, n'allait pas jusqu'à l'ignorance, bien au contraire. Bakounine considérait par exemple que l'on pouvait ratifier la critique de l'économie politique contenue dans Le Capital comme une véritable critique scientifique (qu'il avait d'ailleurs commencé à traduire en russe). On peut d'ailleurs signaler que le premier grand vulgarisateur de l'ouvrage était un ami de Bakounine, le militant italien Carlo Cafiero, dont l'abrégé du Capital s'attira les éloges de Marx et d'Engels ${ }^{33}$. Par la suite, les anarchistes ont pu s'approprier la critique marxienne de l'économie politique comme ils s'appropriaient d'autres connaissances scientifiques utiles pour la propagande et la lutte ${ }^{34}$. Bien entendu, cela implique de considérer qu'il est possible de séparer chez Marx le bon grain de l'économie politique de l'ivraie de la politique et de la philosophie de l'histoire, ce qui n'a rien d'évident.

Enfin, il importe de mentionner les rapprochements qui ont pu être tentés dans l'histoire entre marxisme et anarchisme. Maximilien Rubel, éditeur en français de Marx dans la Bibliothèque de la Pléiade, n'a pas hésité à voir en Marx un « théoricien de l'anarchisme », en insistant notamment sur les écrits suscités chez lui par la Commune de Paris ${ }^{35}$. Daniel Guérin, écrivain et militant communiste libertaire, a pour sa part appelé d'une manière réitérée à une appropriation anarchiste du marxisme ${ }^{36}$. Il faut toutefois souligner que ce furent là des cas assez isolés, au point que certains auteurs marxistes, qui parvenaient à des conclusion proches du communisme anarchiste, se sont toujours refusé à le reconnaître ${ }^{37}$. Il faut enfin faire un sort particulier aux situationnistes qui, dans les années 60, proposèrent un dépassement original de ce qu'ils considéraient comme les unilatéralités respectives du marxisme et de l'anarchisme ${ }^{38}$.

32 Dans sa lettre du 10 octobre 1868 à J. B. Schweitzer, Marx explique ainsi que « l'organisation centralisée [...] est en contradiction avec la nature même des syndicats », qu'elle n'est «pas souhaitable, surtout en Allemagne » où « les ouvriers [...] sont dressés dès l'enfance par la bureaucratie à croire en l'autorité et l'instance supérieure, si bien qu'il faut avant tout qu'ils apprennent à se tirer d'affaire tout seuls ».

33 Carlo Cafiero, Abrégé du Capital de Karl Marx (1878), Marseille, Le Chien rouge, 2013.

34 C'est dans cet esprit que fut votée, lors du Congrès international de Londres constitutif de l'Internationale anarchiste (juillet 1881), une motion recommandant aux militants « de donner un grand poids à l'étude des sciences techniques et chimiques, comme moyen de défense et d'attaque ».

35 Maximilien Rubel, « Marx, théoricien de l'anarchisme », in Marx, critique du marxisme, Paris, Payot, 1974.

36 Daniel Guérin, "Anarchisme et marxisme », in L'Anarchisme, Paris, Gallimard, 1981, et Pour un marxisme libertaire, Paris, Robert Laffont, 1969.

37 C'est le cas notamment d'Anton Pannekoek dont le grand ouvrage Les Conseils ouvriers ne mentionne l'anarchisme que comme une insulte adressée aux ouvriers qui se lancent dans des grèves sauvages (voir Les conseils ouvriers, Paris, Bélibaste, 1971, p. 275).

38 Voir J.-C. Angaut, « Beyond black and red : The Situationists and the legacy of the workers' movement » in Alex Pritchard, Dave Berry, Ruth Kinna, Saku Pinta (ed.), Libertarian Socialism - Politics in Black and Red, Basingstoke, Palgrave Macmillan, 2012, pp. 232-250. 
Fondamentalement, le débat théorique occasionné par le conflit entre anarchisme et marxisme a légué aux mouvements de lutte contre la domination un projet problématique : celui de l'autoémancipation. Pour cette raison, il importe d'apercevoir que la pertinence des critiques anarchistes du marxisme dépasse largement le contexte immédiat dans lequel ces critiques ont été énoncées. Qu'il s'agisse d'identifier le sujet révolutionnaire, de comprendre les conditions sous lesquelles une société débarrassée de l'État est possible ou de parer aux reproductions de la domination au sein même de ces moyens d'émancipation que sont censées constituer les organisations, les problèmes pointés par ces critiques sont encore les nôtres.

Jean-Christophe Angaut 